Reprinted from : CRUSTACEANA, Vol. 21,Part 1,1971

LEIDEN

E. J. BRILL

Article $\mathrm{N}^{\circ} 10$

Contribution $\mathrm{COB} \mathrm{N}^{\circ} 9$

\title{
LE GENRE STAUROSOMA WILL (COPEPODE PARASITE D'ACTINIES) EN MER ROUGE
}

\author{
PAR \\ LUCIEN LAUBIER \\ Centre Océanologique de Bretagne, B.P. 337, Brest 29N, France \\ ET \\ HAJO SCHMIDT \\ Zoologisches Institụt der Universität, Heidelberg, Allemagne
}

- Les représentants du genre Staurosoma Will, 1844 sont des Copépodes gallicoles parasites d'Actinies. Le genre comprend actuellement deux espèces, Staurosoma parasiticum Will, 1844, parasite de Anemonia sulcata Pennant, 1766 (= Actinia viridis) des côtes méditerranéennes (Will, 1844), dans la région du Cap de La Hague (Manche) et le Golfe de Marseille (Caullery \& Mesnil, 1902), et S. caulleryi Okada, 1927, parasite de Nemantbus nitidus (Wassilieff, 1908) (= Sagartia nitida) dans la Baie de Sagami au Japon. De Zulueta (1911) a créé pour ce genre unique la famille des Staurosomidae, famille que des auteurs plus récents suggèrent de rattacher à l'ensemble des Copépodes Chondracanthidae. Une mise au point des connaissances actuelles a été fournie par Bouligand (1966), qui précise les différences entre les deux espèces, mais n'apporte aucune donnée nouvelle. -

Durant le stage organisé par le. "MAMBO"1) pendant l'hiver 1965-1966 à Eilat (Golfe d'Akaba, Mer Rouge), auquel nous avons participé, l'un de nous (H.S.) a trouvé plusieurs spécimens d'un Copépode parasite dans l'Actinie Antbopleura stellula Ehrenberg, 1834. La morphologie caractéristique du parasite, en forme de croix de Lorraine aux bras subégaux, et sa situation dans une galle parfaitement close, ont permis une identification générique rapide: il s'agit d'un représentant du genre Staurosoma, dont nous essayerons de préciser l'espèce après en avoir fait une rapide description.

L'hôte Anthopleura stellula a été récolté sur la plage rocheuse d'Eilat au début de janvier 1966. Les Actinies mesurent de 2 à $4 \mathrm{~cm}$ de hauteur, et le diamètre de la colonne pour une extension normale est de 1 à $1,5 \mathrm{~cm}$. A. stellula vit dans des eaux très superficielles, entre 20 et $50 \mathrm{~cm}$ de profondeur, fixée sous des pierres plates ou dans de petites cavités. Cette espèce est connue depuis l'Ile de Zanzibar jusqu'à Eilat au Nord de la Mer Rouge (des données plus complètes sont fournies par Schmidt, 1970). Sur 62 spécimens récoltés, 6 d'entre eux contenaient des capsules de $0,5 \mathrm{~cm}$ de diamètre environ, formées par une cloison mésentérique

1) Mediterranean Association for Marine Biology and Oçeanography. 
unique (dans un seul cas, la capsule est formée par deux mésentères). Trois hôtes contenaient un seul couple de parasites dans une capsule unique; une Actinie a livré trois capsules contenant chacune un couple de parasites; dans une autre, les parasites étaient encore à l'extérieur du mésentère; enfin, la sixième Actinie contenait une capsule vide.

On peut ainsi reconnaître les stades successifs suivants:

a) Le couple de Copépodes attaque l'endoderme d'un côté d'un mésentère, provoquant probablement la formation de la capsule; la femelle est encore immature.

b) Le couple de Copépodes est enfermé dans la capsule mésentérique; les cordons ovigères existent, blanchâtres: l'un d'eux montre une rangée unique, l'autre plusieurs rangées d'oeufs au début du développement (pl. 1).

c) Le couple de Copépodes est un peu plus grand, toujours enfermé dans une capsule; les cordons ovigères contiennent des oeufs en voie de développement et des nauplius déjà formés (voir Schmidt, 1970, pl. I, B).

d) Il reste une capsule aux parois épaisses, emplie de débris, et ouverte sur l'équateur.

Les capsules sont habituellement situées au milieu de la colonne, sauf dans le cas de l'Actinie contenant trois capsules, qui sont alors situées à différents niveaux. Les parasites de ce spécimen étaient d'ailleurs à divers stades de maturité. Dans la région de la capsule, le mésentère a perdu ses filaments, ainsi qu'une partie du muscle rétracteur. Extérieurement, il n'est pas possible de distinguer une Actinie indemne d'une Actinie infestée.

\section{DESCRIPTION (fig. 1)}

La femelle est recourbée en arc de cercle, et présente une partie concave correspondant à la face dorsale et une partie convexe correspondant à la face ventrale. Une fois étendu, le corps mesure environ $5 \mathrm{~mm}$ de longueur, dimensions inférieures à celles de $S$. parasiticum et de $S$. caulleryi. Les cordons ovigères multisériés sont enroulés autour de la femelle, et le mâle nain fixé sur le segment génital est visible dorsalement. Le corps comporte neuf segments; un premier segment antérieur porte un complexe appendiculaire comprenant deux appendices arrondis, latéro-antérieurs, représentant les antennules, deux autres appendices latéro-postérieurs correspondant aux antennes, enfin deux protubérances, l'une dorsale, l'autre ventrale, situées au niveau des antennes. La protubérance dorsale est de forme conique, dirigée vers l'avant. La protubérance ventrale, de forme hémisphérique, est appliquée du côté postérieur contre une plaque sclérifiée portant une série de fortes soies formant une sorte de brosse. Cette structure a été décrite par Caullery \& Mesnil (1902), et ces auteurs ont également observé en coupes histologiques un très mince oesophage qui peut faire complètement défaut chez certains individus. Il n'existerait pas de bouche chez Staurosoma parasiticum, alors qu'elle semble facile à distinguer chez $S$. caulleryi. Sur les spécimens de Mer Rouge, nous n'avons pu distinguer de bouche, ni par l'observation directe, ni sur coupes histologiques.

Les deux segments suivants, soit les segments th 1 , et th 2 , portent chacun deux 
lobes transversaux tous identiques, constitués de trois articles; le dernier article est subsphérique. Chez S. parasiticum, Caullery \& Mesnil ont observé et figuré à la base de ces lobes, et sur la face ventrale, quatre tubercules; nous n'avons rien remarqué de semblable sur nos individus erythréens. Les lobes correspondants de $S$. caulleryi présentent deux lobules ventraux supplémentaires sur le pénultième et le dernier article.

Les segments thoraciques suivants ( $\mathrm{th}_{3}, \mathrm{th}_{4}$, et th $\mathrm{h}_{5}$ ) sont dépourvus d'appendices transversaux. $T^{T} h_{3}$ et th $h_{4}$ sont semblables; th ${ }_{5}$ est un peu plus grand, et renflé dorsalement. L'ensemble rappelle beaucoup $S$. parasiticum.

Le segment génital porte latéro-dorsalement deux orifices génitaux fortement sclérifiés, d'où sortent les cordons ovigères à l'intérieur desquels les nauplius près d'éclore sont bien visibles. Entre les deux orifices génitaux, soit sur la ligne médiodorsale, le mâle nain est fixé sur le corps de la femelle; lorsqu'il est retiré, on peut observer une boutonnière à bords sclérifiés.

Enfin, les deux derniers segments, qui paraissent presque indivis, sont semblables à leurs homologues chez $S$. parasiticum.
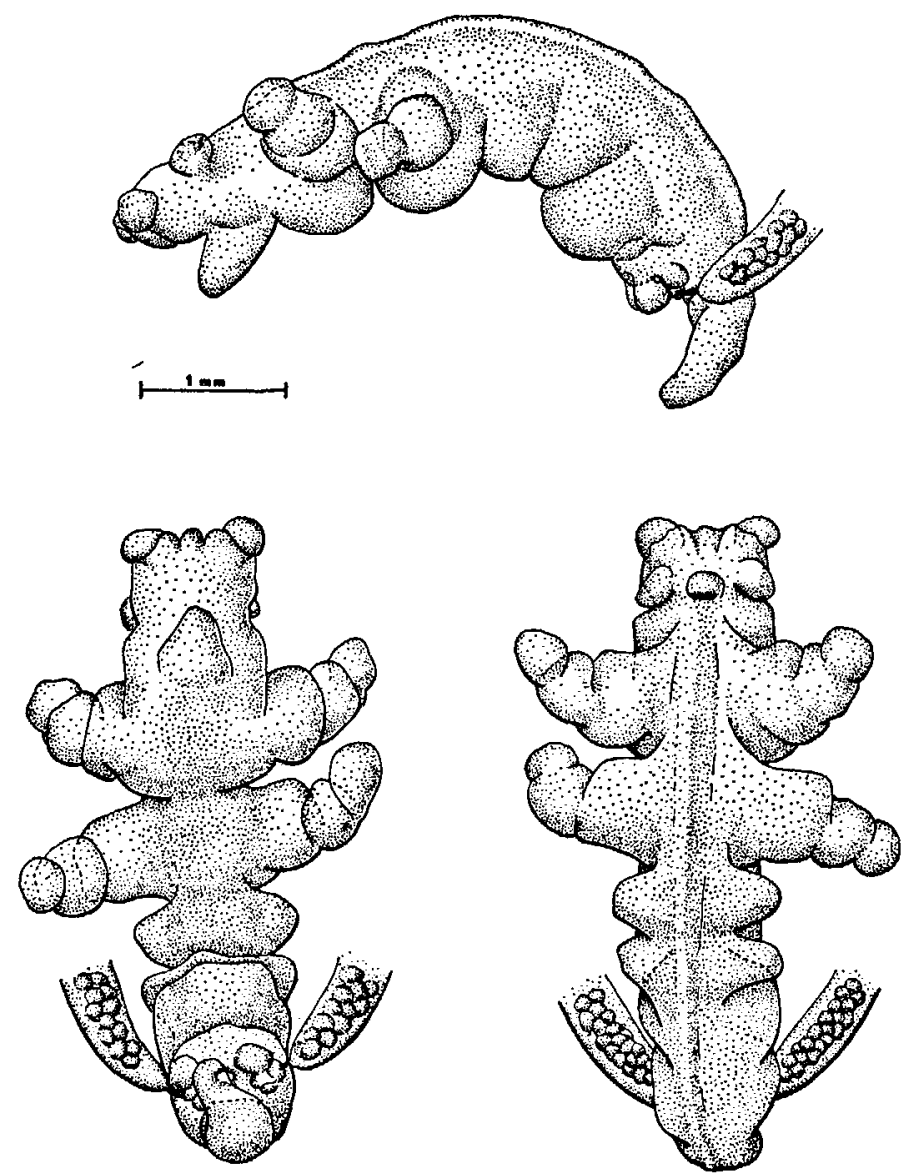

Fig. 1. Staurosoma parasiticum Will, spécimen de Mer Rouge: vues latérale, dorsale (en bas à gauche) et ventrale (en bas à droite). 


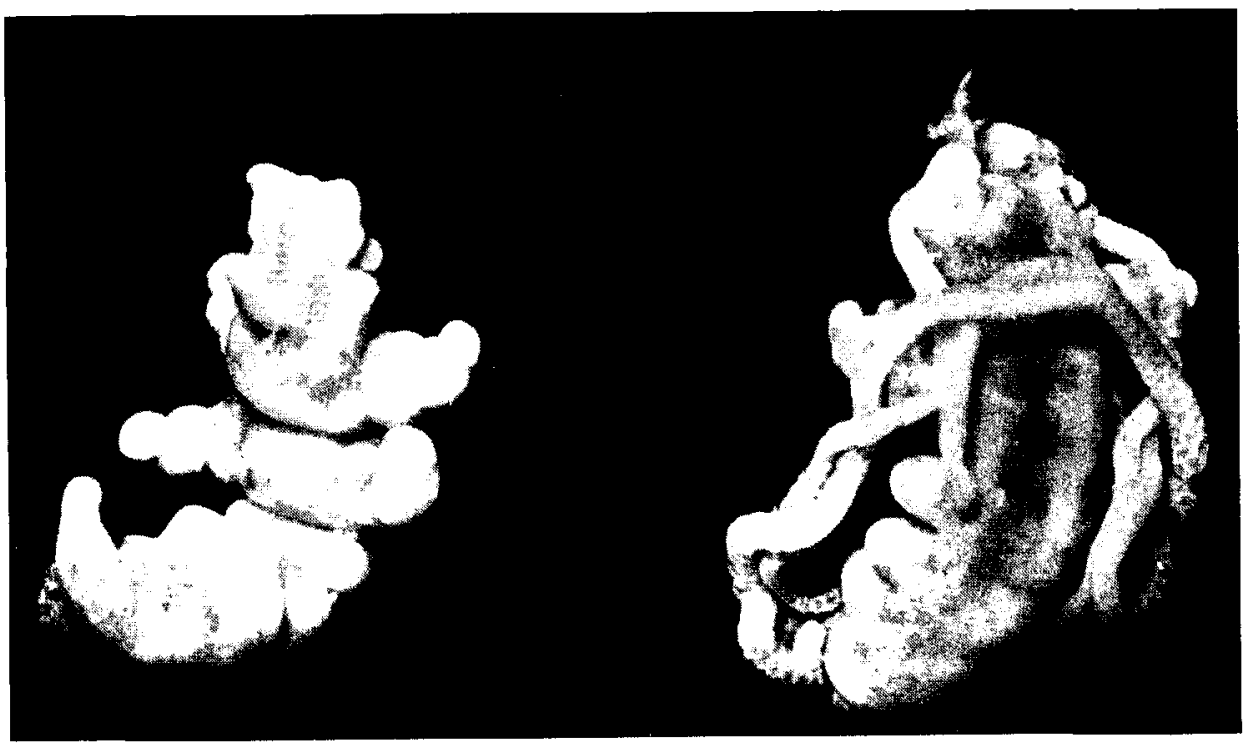

Staurosoma parasiticum Will, spécimens de Mer Rouge: à gauche, femelle en vue latérodorsale, sacs ovigères enlevés; à droite, femelle en vue latéroventrale, avec ses sacs ovigères blanchâtres $(\times 12,5)$. 
Le mâle nain correspond à peu près à la description de Caullery \& Mesnil, ainsi qu'à la figure dessinée par Bouligand (1966) d'après les observations et la figure des auteurs précédents.

\section{DISCUSSION}

S. parasiticum a été récolté à trois reprises (Will, 1844; Caullery \& Mesnil, 1902), toujours dans le même hôte, Anemonia sulcata. On pourrait donc admettre que cette espèce a une spécificité parasitaire étroite, ce qui, joint aux différences biogéographiques, conduirait à reconnaître l'existence d'une espèce particulière pour le parasite du Golfe d'Akaba. Cependant, les critères morphologiques sont très insuffisants pour justifier cette hypothèse. Le seul point par lequel nos spécimens peuvent être distingués de $S$. parasiticum réside dans l'absence de tubercules ventraux à la base des lobes transversaux chez les individus de Mer Rouge. Comptetenu de l'extraordinaire souplesse des téguments de Staurosoma, de leur mode de vie dans des galles fermées, et en l'absence d'autres critères quantitatifs précis, il nous paraît tout à fait prématuré de créer une espèce nouvelle pour la forme de Mer Rouge. La taille plus petite de ces spécimens $(5 \mathrm{~mm}$ contre $25 \mathrm{~mm}$ d'après Caullery \& Mesnil)ne paraît pas non plus devoir être retenue, dans la mesure où les conditions thermiques propres au Golfe d'Akaba peuvent accélérer l'acquisition de la maturité sexuelle. Il faut également souligner que les deux hôtes n'ont pas la même taille: Anthopleura stellula est plus petite que Anemonia sulcata.

Nous estimons donc préférable d'identifier les spécimens de Mer Rouge à $S$. parasiticum, tout en remarquant que du point de vue biogéographique, il eût été plus naturel de trouver en Mer Rouge l'espèce japonaise (mais des raisons morphologiques importantes ne permettent pas le doute à cet égard). D'autre part, cette conclusion revient à élargir la spécificité de $S$. parasiticum, qui paraît susceptible de vivre dans deux hôtes différents, Anemonia sulcata Pennant, 1766 et Anthopleura stellula Ehrenberg, 1834. Enfin, il est clair que des progrès décisifs dans nos connaissances ne pourraient provenir que de l'étude complète du développement, seule façon d'évaluer la valeur de l'hypothèse avancée par Caullery \& Mesnil et reprise par Okada, selon laquelle Staurosoma est à rattacher aux Chondracanthidae.

\section{SUMMARY}

Staurosoma, a genus of Copepoda parasitic in actinians, is recorded for the first time from the Red Sea (Eilat) and from a new host, Antbopleura stellula. Morphological study indicates that this parasite is identical to the European species, $S$. parasiticum Will, a rather surprising conclusion from a biogeographical point of view.

\section{BIBLIOGRAPHIE}

Bouligand, Y, 1966. Recherches récentes sur les Copépodes associés aux Anthozoaires. In: The Cnidaria and their evolution. Symp. zool. Soc. London, 16: 267-306. (Academic Press).

CAullary, M. \& F. Mesnil, 1902. Sur Staurosoma parasiticum Will, Copépode gallicole, parasite d'une Actinie. C. r. hebd. Séanc. Acad. Sc., Paris, 134: 1314-1317.

OKadA, Y. K., 1927. Staurosoma, Copépode parasite d'une Actinie: description de Staurosoma caulleryi sp. n. Annotnes zool. jap., 11: 173-183. 
Schmidt, H., 1970. On Anthopleura stellula Ehrenberg, 1834 (Actiniaria, Actiniidae) and its reproduction by transverse fission. Marine Biology, 5: 245-255.

WILL, F., 1844. Uber Staurosoma, einen in den Aktinien lebenden Schmarotzer. Arch. Naturgesch., 10 (1): $337-343$.

ZuluetA, A. DE, 1911. Los copepodos parasitos de los celentereos. Mem. R. Soc. esp. Hist. nat.. 7: 5 -58. 